\title{
Comparison between New-Onset and Old-Diagnosed Type 2 Diabetes with Ketosis in Rural Regions of China
}

\author{
Shichun Du, ${ }^{1}$ Xia Yang, ${ }^{2}$ Degang Shi, ${ }^{2}$ and Qing Su${ }^{1}$ \\ ${ }^{1}$ Department of Endocrinology, Xinhua Hospital, Shanghai Jiaotong University School of Medicine, Shanghai 200092, China \\ ${ }^{2}$ Department of Endocrinology, Baoshan People's Hospital, Yunnan 678000, China \\ Correspondence should be addressed to Qing Su; suqingxinhua@163.com
}

Received 25 October 2015; Revised 30 December 2015; Accepted 12 January 2016

Academic Editor: Franco Veglio

Copyright (c) 2016 Shichun Du et al. This is an open access article distributed under the Creative Commons Attribution License, which permits unrestricted use, distribution, and reproduction in any medium, provided the original work is properly cited.

Objectives. Type 2 diabetes (T2D) with ketosis was common because of late diagnosis and lacking adequate treatment in rural regions of China. This study aimed to provide the data of T2D with ketosis among inpatients in a south-west border city of China. Methods. Data of 371 patients of T2D with ketosis who were hospitalized between January 2011 and July 2015 in Baoshan People's Hospital, Yunnan, China, were analyzed. New-onset and old-diagnosed T2D patients presenting with ketosis were compared according to clinical characteristics, laboratory results, and chronic diabetic complications. Results. Overall, the blood glucose control was poor in our study subjects. Male predominated in both groups (male prevalence was $68 \%$ in new-onset and $64 \%$ in old-diagnosed groups). Overweight and obesity accounted for 50\% in new-onset and $46 \%$ in old-diagnosed cases. Inducements of ketosis were $13.8 \%$ in new-onset and $38.7 \%$ in old-diagnosed patients. Infections were the first inducements in both groups. The prevalence of chronic complications of diabetes was common in both groups. Conclusions. More medical supports were needed for the early detection and adequate treatment of diabetes in rural areas of China.

\section{Introduction}

The prevalence of type 2 diabetes (T2D) in rural regions is rising rapidly and has been a great challenge to the local health care programs [1]. In China, large parts of diabetic individuals lived in rural areas, where resources to screen and treat diabetes and its complications were limited [13]. Patients with diabetes usually delayed their adequate treatment due to late screen and diagnosis. Ketosis was frequently presented in these patients. So far, the situations of type 2 diabetes presenting with ketosis prior to admission in the south-west rural region were unclear.

Ketosis is a symbol of acute disorder of glucose and lipids metabolism in diabetic patients. Ketosis or ketoacidosis has been regarded as trait of type 1 diabetes (T1D). However, patients with T2D were susceptible to ketosis or ketoacidosis under extreme hyperglycemia. Because of backward in economy, quite a few patients did not get duly treatment until severe hyperglycemia or diabetic complications occurred. T2D patients with ketosis were frequently seen at clinic in rural areas. Our present study was to investigate the prevalence and characteristics of T2D presenting with ketosis in rural areas aiming to suggest more efforts for the prevention and control of diabetes in rural areas of China.

\section{Methods}

We performed a retrospective analysis of T2D with ketosis in patients admitted to the Endocrinology Department of Baoshan People's Hospital of Yunnan Province between January 2011 and July 2015. Overall, data of 391 T2D presenting with ketosis inpatients aged 12 years or older were enrolled. Nine patients were excluded because of surgery, pregnancy, trauma, secondary diabetes, or pancreatic exocrine diseases. 11 patients were excluded because of complicated with unconsciousness. Thus, the other 371 patients (245 males and 126 females) were allocated to the analysis. According to the diabetic duration, patients were divided into new-onset or old-diagnosed group. New-onset group included those with 
new diagnosed diabetes or no more than 6 months after onset. Old-diagnosed group were patients of previously diagnosed T2D presenting with ketosis at this admission. Diabetes were defined in accordance with diagnostic criteria of American Diabetes Association [4]. T2D was diagnosed if patients had clinical and metabolic features including overweight or obesity, more than 40 years age at onset, and obvious diabetes family history in combination with preserved $\beta$-cell function (fasting C-peptide of more than $0.33 \mathrm{nmol} / \mathrm{L}$ ) and negative $\beta$-cell autoantibodies. T2D with ketosis was diagnosed if patients met the criteria of T2D and had positive urinary ketone body results this time on admission. Urinary ketone body positive was diagnosed if urinary acetoacetate increased over $15 \mathrm{mg} / \mathrm{dL}$ by using sodium nitroprusside method. Acidosis was diagnosed if an arterial $\mathrm{pH}$ of less than 7.35 and/or blood bicarbonate level of less than $15 \mathrm{mmol} / \mathrm{L}$ in the context of hyperglycemia and ketonuria was found. Overweight and obesity were defined if body mass index (BMI) $\geq 24$, which was set forth by Chinese guidance of overweight and obesity in adults [5]. Data of demographic and clinical characteristics including age, gender, height, weight, BMI, blood pressure, and diabetic family history were collected. Laboratory parameters including plasma glucose, $\mathrm{HbAlc}, \mathrm{C}$ peptide, total cholesterol, and triglycerides were recorded. Blood samples were performed at the time on admission after an overnight fast. The chronic diabetic complications including peripheral neuropathy, retinopathy, diabetic foot, and persistent microalbuminuria that were evaluated by trained physicians during the hospitalization.

All of the data were analyzed by JMP 9.0 (SAS Institute, Cary, NC). The ANOVA or Mann-Whitney test analysis was used to evaluate the differences in continuous variables. Chisquared statistical analysis was used for categorical variables. Continuous data in the text and tables were expressed as means \pm SD. Categorical variables were expressed by either absolute numbers or percentages. Statistical significance was defined as two-tailed $P$ values $<0.05$.

\section{Results}

Table 1 showed the clinical characteristics of the new-onset and old-diagnosed T2D with ketosis patients. Among the total 371 subjects, 211 of the patients (57\%) were classified as new-onset, while 160 (43\%) were old-diagnosed T2D with ketosis. The subjects of new-onset group had an average duration of 1.7 months with weight loss of $7.3 \mathrm{~kg}$, while the old-diagnosed group had an average diabetic duration of about 6.3 years with weight loss of $9.2 \mathrm{~kg}$. Subjects of newonset group were younger $(47 \pm 12$ versus $53 \pm 13 \mathrm{yr}, P=0.001)$ and more overweight (50\% versus $46 \%, P=0.009$ ) than those old-diagnosed T2D with ketosis. Family history of diabetes was similar $(P=0.79)$ between new-onset $(35 \%)$ and olddiagnosed (34\%) group. The blood pressure of the analyzed subjects was not significantly different between two groups.

Table 2 showed the biochemical characteristics of the new-onset and previously diagnosed T2D patients presenting with ketosis. Fasting plasma glucose was significantly elevated in both new-onset $(17.0 \pm 6 \mathrm{mmol} / \mathrm{L})$ and old-diagnosed $(16.4 \pm 7 \mathrm{mmol} / \mathrm{L}, P=0.49)$ group. $2 \mathrm{~h}$ plasma glucose was
TABLE 1: Clinical characteristics of the new-onset and old-diagnosed T2D with ketosis patients.

\begin{tabular}{lccc}
\hline & \multicolumn{3}{c}{ T2D with ketosis } \\
& New-onset & Old-diagnosed & $P$ \\
\hline Subjects (total \%) & $211(57 \%)$ & $160(43 \%)$ & - \\
Duration (months) & $1.73 \pm 2.5$ & $75.36 \pm 63.2$ & - \\
Weight reduction (kg) & $7.31 \pm 4.45$ & $9.23 \pm 6.34$ & 0.03 \\
Age (yr) & $47 \pm 12$ & $53 \pm 13$ & 0.001 \\
Gender (male \%) & 68 & 64 & 0.15 \\
Diabetic family history (\%) & 35 & 34 & 0.79 \\
Height (cm) & $163 \pm 9$ & $160 \pm 10$ & 0.01 \\
Weight (kg) & $66 \pm 14$ & $62 \pm 15$ & 0.04 \\
Body mass index (kg/m $\left.{ }^{2}\right)$ & $25 \pm 4$ & $24 \pm 5$ & 0.28 \\
Overweight or obese $(\%)$ & 50 & 46 & 0.009 \\
Systolic pressure (mmHg) & $119 \pm 20$ & $122 \pm 21$ & 0.25 \\
Diastolic pressure $(\mathrm{mmHg})$ & $79 \pm 12$ & $78 \pm 13$ & 0.61 \\
\hline
\end{tabular}

TABLE 2: Laboratory results of the new-onset and old-diagnosed T2D with ketosis patients.

\begin{tabular}{lccc}
\hline & \multicolumn{3}{c}{ T2D with ketosis } \\
& New-onset & Old-diagnosed & $P$ \\
\hline $\begin{array}{l}\text { Fasting glucose } \\
\text { (mmol/L) }\end{array}$ & $17.0 \pm 6$ & $16.4 \pm 7$ & 0.49 \\
$\begin{array}{l}\text { Postprandial 2-hour } \\
\text { glucose (mmol/L) }\end{array}$ & $24.2 \pm 8$ & $24.1 \pm 9$ & 0.86 \\
$\begin{array}{l}\text { Fasting C-peptide } \\
\text { (nmol/L) }\end{array}$ & $0.69 \pm 0.11$ & $0.58 \pm 0.17$ & 0.60 \\
$\begin{array}{l}\text { Postprandial 2-hour } \\
\text { C-peptide (nmol/L) }\end{array}$ & $2.58 \pm 1.1$ & $1.26 \pm 0.9$ & 0.02 \\
$\begin{array}{l}\text { Hemoglobin Alc (\%) } \\
\text { Fructosamine (mmol/L) }\end{array}$ & $13.2 \pm 3.2$ & $13.7 \pm 4.1$ & 0.34 \\
$\begin{array}{l}\text { Ketoacidosis (\%) } \\
\text { Total cholesterol } \\
\text { (mmo/L) }\end{array}$ & 6.6 & $3.61 \pm 0.8$ & 0.11 \\
$\begin{array}{l}\text { Triglyceride (mmo/L) } \\
\text { Serum creatinine } \\
(\mu \text { mol/L) }\end{array}$ & $4.4 \pm 4.0$ & $3.7 \pm 4.2$ & 0.004 \\
\begin{tabular}{l} 
Serum albumin (g/L) \\
\hline
\end{tabular} & $41.4 \pm 7$ & $40.1 \pm 6$ & 0.190001 \\
\hline
\end{tabular}

also similarly elevated $(P=0.86)$ between new-onset $(24.2 \pm$ $8 \mathrm{mmol} / \mathrm{L})$ and old-diagnosed $(24.1 \pm 9 \mathrm{mmol} / \mathrm{L})$ group. Fasting C-peptide levels were similar $(P=0.60)$ between new-onset $(0.69 \pm 0.11 \mathrm{nmol} / \mathrm{L})$ and old-diagnosed $(0.58 \pm$ $0.17 \mathrm{nmol} / \mathrm{L}$ ) group. However, $2 \mathrm{~h}$ C-peptide was significantly higher $(P=0.02)$ in new-onset $(2.58 \pm 1.1 \mathrm{nmol} / \mathrm{L})$ than old-diagnosed $(1.26 \pm 0.9 \mathrm{nmol} / \mathrm{L})$ group. The hemoglobin $\mathrm{Alc}(\mathrm{HbAlc})$ and fructosamine were not different between the two groups. More patients in the old-diagnosed group (19.4\%) suffered from acidosis than new-onset group (6.6\%). Total cholesterol $(5.9 \pm 1.7$ versus $4.9 \pm 1.8 \mathrm{mmol} / \mathrm{L}$, resp., in new-onset and old-diagnosed group, $P<0.0001)$ and triglyceride $(4.4 \pm 4.0$ versus $3.7 \pm 4.2 \mathrm{mmol} / \mathrm{L}$, resp., in newonset and old-diagnosed group, $P=0.004$ ) were significantly higher in new-onset than in old-diagnosed group. 


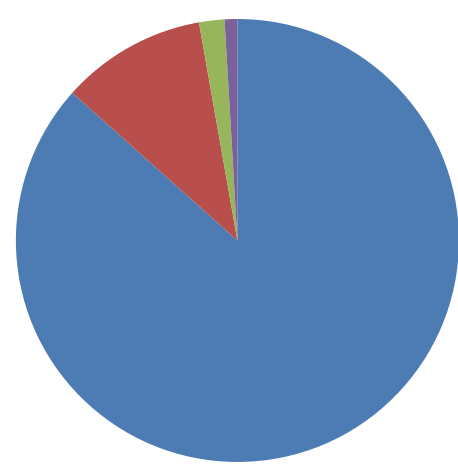

No inducement

- Respiratory system infection

Urinary system infection

Other

(a) New-onset T2D with ketosis

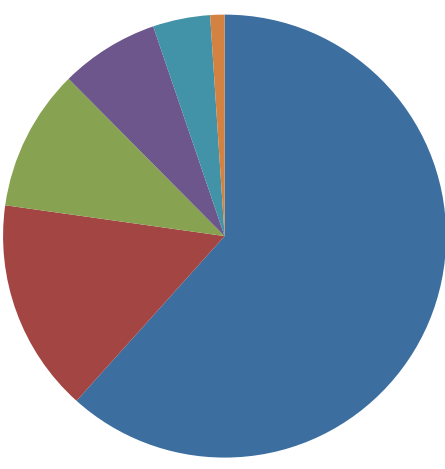

No inducement

- Respiratory system infection

- Urinary system infection

Digestive system infection

Noncompliance with drug therapy

(b) Old-diagnosed T2D with ketosis

FIgURE 1: Potential factors precipitating ketosis in T2D patients.

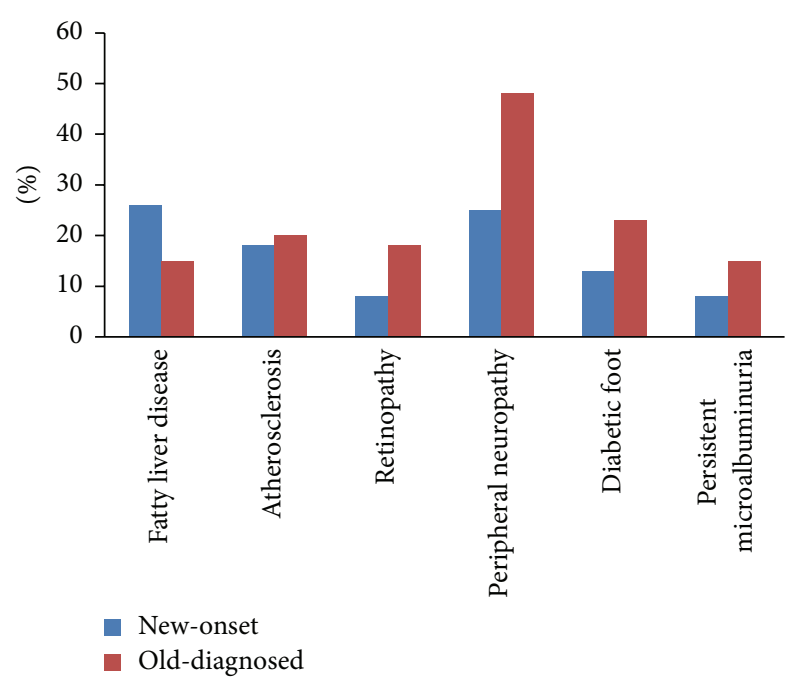

FIGURE 2: Prevalence of diabetic chronic complications in new-onset and old-diagnosed T2D with ketosis.

Figure 1 showed the potential factors precipitating ketosis in T2D patients. Those new-onset patients showed a lower incidence of predisposing factors than previously diagnosed T2D patients presenting with ketosis $(13.8 \%$ versus $38.7 \%, P<$ 0.001 ). The potential triggers for subjects in T2D with ketosis were varied. Among them, infections were predominant in both groups. The prevalence of respiratory system infection was $10.3 \%$ in new-onset and $14.9 \%$ in old-diagnosed group. Urinary system infection was $1.8 \%$ in new-onset and $9.9 \%$ in old-diagnosed group.

Figure 2 showed the prevalence of chronic diabetic complications in new-onset and previously diagnosed T2D with ketosis. The patients with new-onset had higher $(P<0.0001)$ prevalence of fatty liver disease $(26.2 \%)$ compared with those of old-diagnosed (15.1\%) group, while the prevalence of most chronic complications was higher in old-diagnosed group than new-onset group, including retinopathy $(8.1 \%$ versus $17.8 \%$, resp., in new-onset and old-diagnosed group, $P<0.0001)$, peripheral neuropathy $(25.3 \%$ versus $47.6 \%$, resp., in new-onset and old-diagnosed group, $P<0.0001$ ), diabetic foot (13.3\% versus $23.2 \%$, resp., in new-onset and old-diagnosed group, $P<0.0001)$, and persistent microalbuminuria $(8.2 \%$ versus $15.1 \%$, resp., in new-onset and olddiagnosed group, $P<0.0001)$. No significant differences $(P=$ $0.45)$ in atherosclerosis were found in new-onset $(18.0 \%)$ and old-diagnosed (20.1\%) group.

\section{Discussion}

In this study, we showed the characteristic of T2D with ketosis in rural areas of south-west part of China. It was concluded from the study that the control of $\mathrm{T} 2 \mathrm{D}$ was poor in rural area, as reflected not only by extremely elevated HBAlc and plasma glucose performed once on admission but also by high prevalence of chronic diabetic complications evaluated during hospitalization. There were limited data about the prevalence of ketosis in type 2 diabetes population. In a study of 134 Chinese patients with severe hyperglycemia, there were 24 patients diagnosed with ketosis (17.9\%) and 6 patients diagnosed with ketoacidosis (4.5\%) [6]. The data indicated the high prevalence of ketosis in T2D with severe hyperglycemia.

Subjects of new-onset and old-diagnosed T2D with ketosis group had similarities and differences at clinical manifestations. Consistent with previous studies, we found a predominance of male proportion in the study subjects [711]. This prevalence was more obvious in those of new-onset group. The underlying mechanisms of male predominance in T2D with ketosis were unknown and needed further investigation. Differences of body fat distribution and sex hormone were supposed to be factors contributing to the gender difference $[12,13]$. 
In our study, the prevalence of hyperlipidemia was high in both new-onset and old-diagnosed subject, which were true with many previous studies [11, 14-16]. Some investigators have reported that acute elevation of free fatty acid levels in the circulation increased insulin resistance and impaired $\beta$ cell function $[17,18]$. In our study subjects, the prevalence of hyperlipidemia was $60 \%$ in subjects of new-onset group and $29 \%$ in old-diagnosed group. We analyzed our data and found that hyperlipidemia was common in the overweight diabetic subjects. BMI of the enrolled patients positively correlated with both triglyceride and total cholesterol level (data not shown). The phenomenon further confirmed the view that hyperlipidemia played substantially roles underlying trigger of ketosis especially in new-onset diabetes. Therefore, interventions for prevention and treatment of overweight and hyperlipidemia were essential in T2D patients.

Infections were the most common precipitating factors for ketosis in T2D patients, which was an important point distinguishing T2D from T1D. In patients with T1D, noncompliance with insulin therapy was considered as first reason for precipitating ketosis $[14,19]$. In our old-diagnosed T2D subjects, varied infections accounted for about $40 \%$ of the total precipitating factors, indicating that infections were the important precipitating factors leading to ketosis. However, in new-onset ketosis subjects, most cases were nonprovoked ketosis. The discrepancy in prevalence of precipitating factors might indicate the complexity and heterogeneity of T2D with ketosis. More clinical epidemiological surveys need to be conducted to clarify the heterogeneity in T2D with ketosis.

In our study subjects, patients had a higher incidence of diabetic chronic complications, especially in those previously diagnosed T2D patients. It was shown by literatures [20] that those who came for regular follow-up diabetic treatment had a lower incidence of retinopathy and nephropathy compared to those who had irregular follow-up. Since T2D and most of the chronic complications were silent at first, patients usually did not come to the clinic for routine examination until they developed severe complications of diabetes in rural areas [20]. As a result, most patients were complicated with severe chronic complications at their fist diabetic diagnosis. However, late treatments of diabetes and its complications were often associated with higher costs to the affected patient $[1,20]$. Therefore, it was reasonable to suggest detection and adequate treatment at an earlier stage, by which the excess cost due to treatment of diabetes complications might be minimized.

There were some limitations to the present study. First, we did not perform plasma ketosis test which was more sensitive and specific in detecting ketosis; however, urinary ketone body measurements were widely acceptable in hospitals for diagnosing ketosis. Second, we did not measure the body fat distribution which was a potential and important parameter for explaining gender difference in T2D with ketosis. Third, the single-center study restricted our ability to assess the overall characteristics of T2D with ketosis in rural areas. Further large cohorts and prospective follow-up studies were needed to clarify the characteristics of T2D with ketosis in south-west rural parts of China.
In summary, the study shows the characteristics of T2D with ketosis in adults of rural areas in south-west part of China. T2D with ketosis is a severe disorder of glucose and lipids metabolism and prone to acquire chronic complications. Thus, there is an urgent and important need for the implementation of effective screening, health knowledge promotion, and control of diabetes.

\section{Conflict of Interests}

The authors state that they have no conflict of interests.

\section{Acknowledgments}

The study was supported by the research grant of Shanghai municipal commission of health and family planning, no. 20144Y0140, and the research grant of cooperation between medicine and engineering techniques of Shanghai Jiaotong University, no. YG2015QN43.

\section{References}

[1] R. Unnikrishnan and V. Mohan, "Why screening for type 2 diabetes is necessary even in poor resource settings," Journal of Diabetes and its Complications, vol. 29, no. 7, pp. 961-964, 2015.

[2] Y. Xu, L. Wang, J. He, Y. Bi, M. Li et al., "Prevalence and control of diabetes in Chinese adults," The Journal of the American Medical Association, vol. 310, no. 9, pp. 948-959, 2013.

[3] D. Hu, P. Fu, J. Xie et al., "Increasing prevalence and low awareness, treatment and control of diabetes mellitus among Chinese adults: the InterASIA study," Diabetes Research and Clinical Practice, vol. 81, no. 2, pp. 250-257, 2008.

[4] American Diabetes Association, "Diagnosis and classification of diabetes mellitus," Diabetes Care, vol. 27, supplement 1, pp. S5-S10, 2004.

[5] C. Chen and F. C. Lu, "The guidelines for prevention and control of overweight and obesity in Chinese adults," Biomedical and Environmental Sciences, vol. 17, supplement, pp. 1-36, 2004.

[6] X. Cao, X. Zhang, Y. Xian et al., "The diagnosis of diabetic acute complications using the glucose-ketone meter in outpatients at endocrinology department," International Journal of Clinical and Experimental Medicine, vol. 7, no. 12, pp. 5701-5705, 2014.

[7] W. E. Winter, N. K. Maclaren, W. J. Riley, D. W. Clarke, M. S. Kappy, and R. P. Spillar, "Maturity-onset diabetes of youth in black Americans," The New England Journal of Medicine, vol. 316, no. 6, pp. 285-291, 1987.

[8] A. Piñero-Piloña, P. Litonjua, L. Aviles-Santa, and P. Raskin, "Idiopathic type 1 diabetes in Dallas, Texas: a 5-year experience," Diabetes Care, vol. 24, no. 6, pp. 1014-1018, 2001.

[9] J.-F. Louet, S. B. Smith, J.-F. Gautier et al., "Gender and neurogenin3 influence the pathogenesis of ketosis-prone diabetes," Diabetes, Obesity and Metabolism, vol. 10, no. 10, pp. 912-920, 2008.

[10] K. Yamada and K. Nonaka, "Diabetic ketoacidosis in young obese Japanese men," Diabetes Care, vol. 19, article 671, 1996.

[11] G. Goodstein, A. Milanesi, and J. E. Weinreb, "Ketosis-prone type 2 diabetes in a veteran population," Diabetes Care, vol. 37 , no. 4, pp. e74-e75, 2014. 
[12] K. Blouin, A. Boivin, and A. Tchernof, "Androgens and body fat distribution," Journal of Steroid Biochemistry and Molecular Biology, vol. 108, no. 3-5, pp. 272-280, 2008.

[13] X. Wang and H. Tan, "Male predominance in ketosis-prone diabetes mellitus," Biomedical Reports, vol. 3, no. 4, pp. 439-442, 2015.

[14] H. Tan, Y. Zhou, and Y. Yu, "Characteristics of diabetic ketoacidosis in Chinese adults and adolescents-a teaching hospitalbased analysis," Diabetes Research and Clinical Practice, vol. 97, no. 2, pp. 306-312, 2012.

[15] G. E. Umpierrez, D. Smiley, G. Robalino, L. Peng, A. R. Gosmanov, and A. E. Kitabchi, "Lack of lipotoxicity effect on $\beta$ cell dysfunction in ketosis-prone type 2 diabetes," Diabetes Care, vol. 33, no. 3, pp. 626-631, 2010.

[16] M. Zhang, Y. Li, W. Cui et al., "The clinical and metabolic characteristics of young-onset ketosis-prone type 2 diabetes in China," Endocrine Practice, vol. 21, no. 12, pp. 1364-1371, 2015.

[17] S. Bevilacqua, R. Bonadonna, G. Buzzigoli et al., "Acute elevation of free fatty acid levels leads to hepatic insulin resistance in obese subjects," Metabolism, vol. 36, no. 5, pp. 502-506, 1987.

[18] G. Boden, X. Chen, J. Rosner, and M. Barton, "Effects of a 48-h fat infusion on insulin secretion and glucose utilization," Diabetes, vol. 44, no. 10, pp. 1239-1242, 1995.

[19] L. Barski, R. Nevzorov, I. Harman-Boehm et al., "Comparison of diabetic ketoacidosis in patients with type-1 and type- 2 diabetes mellitus," The American Journal of the Medical Sciences, vol. 345, no. 4, pp. 326-330, 2013.

[20] R. M. Anjana, C. S. Shanthirani, R. Unnikrishnan et al., "Regularity of follow-up, glycemic burden, and risk of microvascular complications in patients with type 2 diabetes: a 9-year followup study," Acta Diabetologica, vol. 52, pp. 601-609, 2015. 


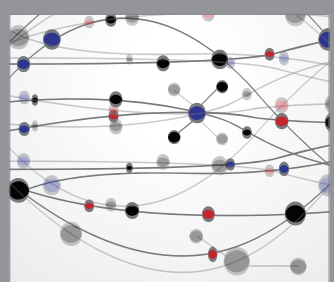

The Scientific World Journal
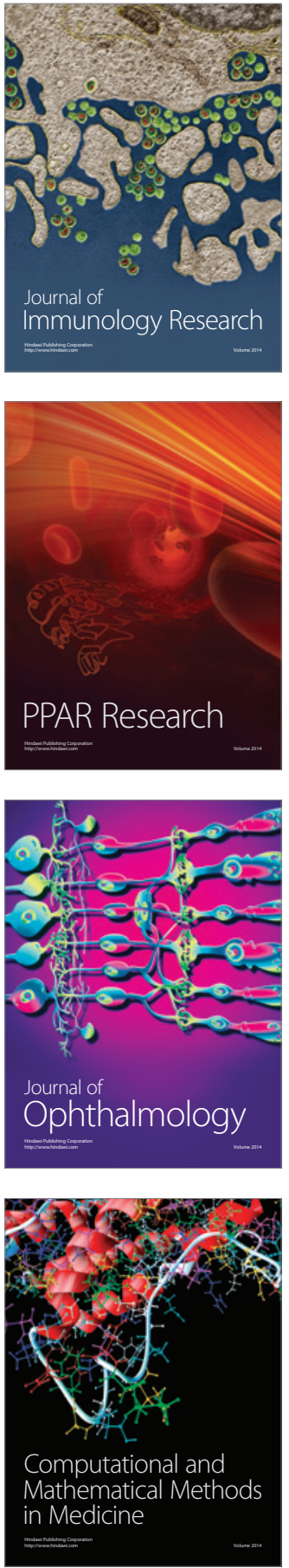

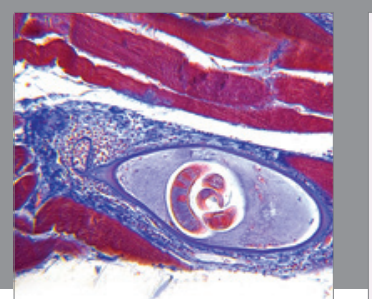

Gastroenterology Research and Practice

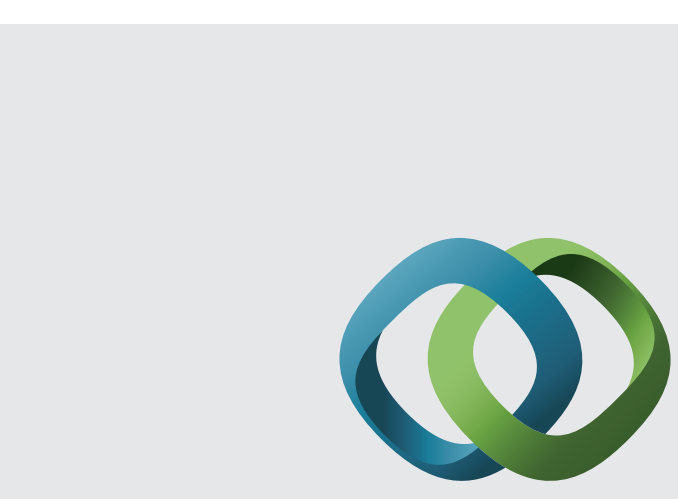

\section{Hindawi}

Submit your manuscripts at

http://www.hindawi.com
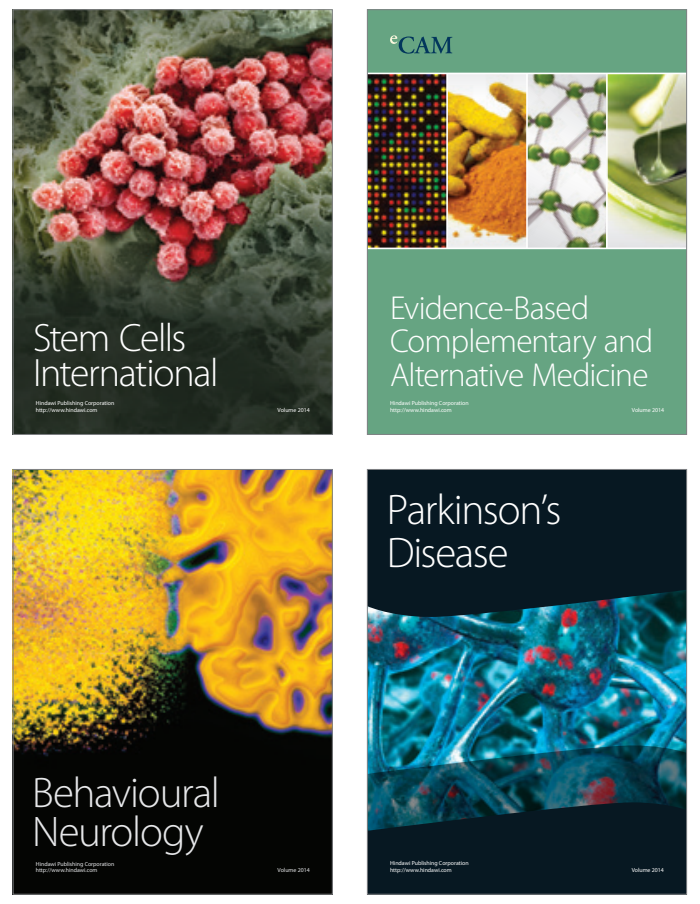
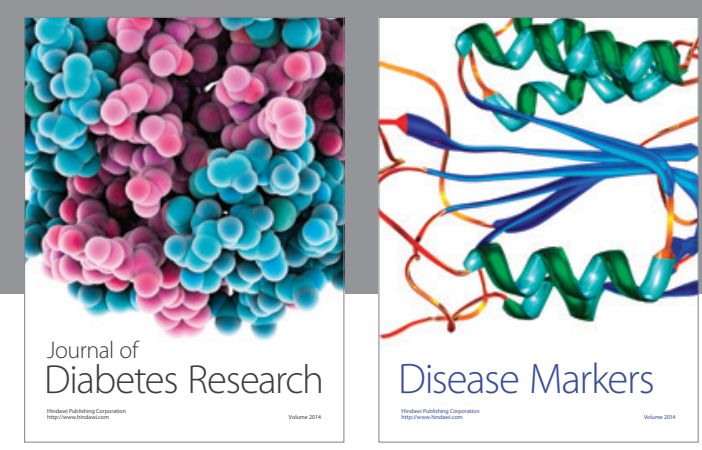

Disease Markers
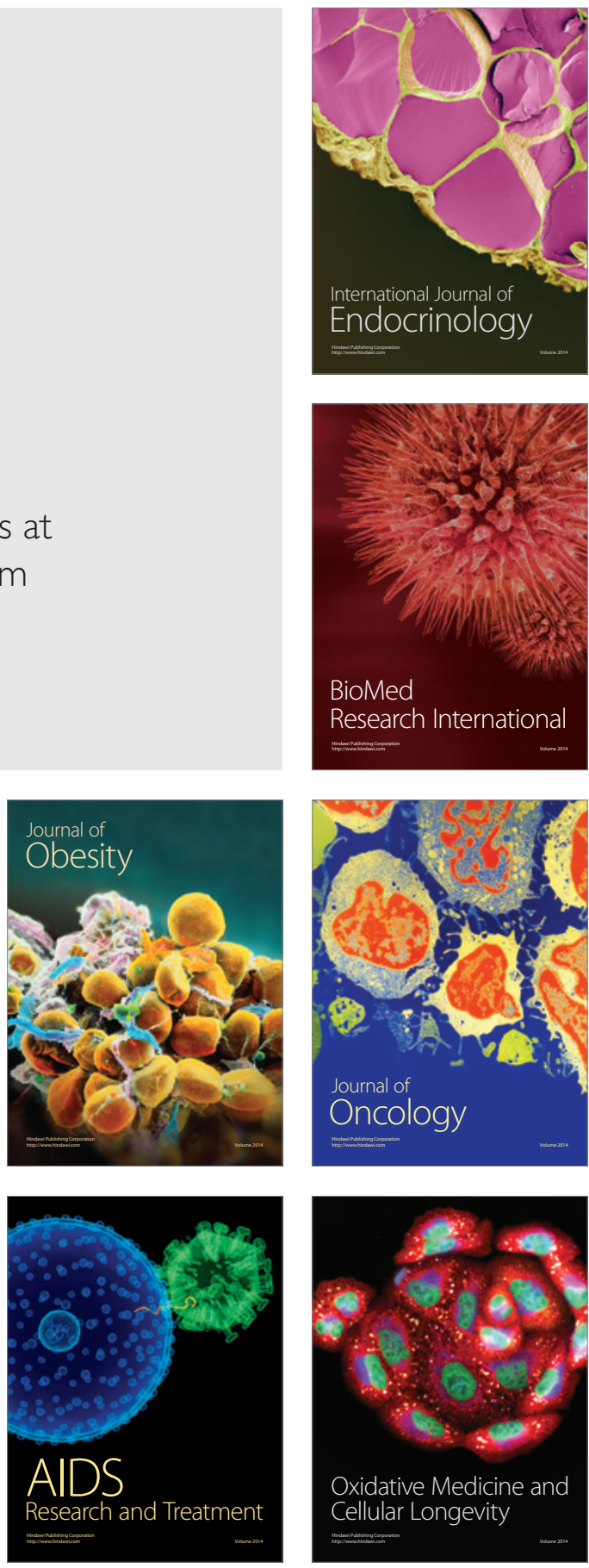